\title{
A LOFAR survey of spatially resolved ultra steep spectrum sources
}

\author{
Leah K. Morabito* \\ Leiden Observatory, Leiden University, P.O. Box 9513, 2300 RA Leiden, The Netherlands \\ E-mail: morabitodstrw. leidenuniv.nl
}

\section{Adam Deller, Javier Moldón}

Netherlands Institute for Radio Astronomy (ASTRON), Postbus 2, NL-7990 AA Dwingeloo, the Netherlands

\section{Raymond Oonk, George Miley, Huub Röttgering}

Leiden Observatory, Leiden University, P.O. Box 9513, 2300 RA Leiden, The Netherlands

\begin{abstract}
The correlation between radio spectral steepness and redshift has been successfully used to find very high redshift radio galaxies. However, the origin of this correlation is still unknown. Understanding particle acceleration processes is fundamental to explaining the correlation. The ultrasteep spectra of high redshift radio sources means they will be bright at low frequencies. The low frequency regime is also where spectral turnovers or cutoffs will be observed due to different physical mechanisms. Thus the new Low Frequency Array (LOFAR) is an ideal instrument for observing these sources. As part of an ongoing survey, we use international baselines to measure the low-frequency spectra $(<200 \mathrm{MHz})$ and their spatial distributions along the jets of 11 bright extended steep spectrum high redshift radio sources. We will present early results from our LOFAR low band antenna survey of ultra-steep spectrum radio galaxies, including complementary high band antenna images of one of the targets. From this, we will measure the shape of the integrated spectra at low frequencies and determine whether the spectra change over these spatially resolved sources, thereby constraining particle acceleration processes. These objects are also candidates for searches for carbon radio recombination lines, the detection of which would provide a unique diagnostic for constraining the physical properties of cold gas in high redshift radio galaxies.
\end{abstract}

EXTRA-RADSUR2015 (*)

20-23 October 2015

Bologna, Italy

(*) This conference has been organized with the support of the Ministry of Foreign Affairs and International Cooperation, Directorate General for the Country Promotion (Bilateral Grant Agreement ZA14GR02 - Mapping the Universe on the Pathway to SKA)

${ }^{*}$ Speaker. 


\section{Introduction}

High redshift radio galaxies (HzRGs) are distant massive galaxies that have spectacular jets of relativistic plasma often extending far beyond the optical host galaxy. High redshift here is taken to be $z \gtrsim 2$. There are several defining characteristics of HzRGs aside from their extended, powerful $\left(>10^{60} \mathrm{ergs}\right.$ ) radio jets. They are often associated with huge haloes of ionized gas, seen via Lyman$\alpha$ emission (Kurk et al., 2000). HzRGs are surrounded by overdensities of galaxies, indicating they are in proto-cluster environments (e.g., Cooke et al., 2014). An alignment is observed between the radio jets and optical star forming regions outside of the host galaxy (e.g., Bicknell et al., 2000; Pentericci et al., 1999; Chambers et al., 1987), suggesting that the radio jets can induce star formation.

While much is known about HzRGs, there are still open questions (see Miley \& De Breuck, 2008, for a review). One of the most pressing questions given by Miley \& De Breuck (2008) is:

"What is the particle acceleration mechanism in the relativistic plasma and why do HzRGs have much steeper radio spectra than nearby radio sources?"

This question is illustrated by Figure 1, which shows the remarkable correlation between the radio spectral index $\left(\alpha ; S \propto v^{-\alpha}\right)$ and the redshift $(z)$ of a source (Blumenthal \& Miley, 1979; Tielens et al., 1979). The fundamental cause of this $z-\alpha$ relationship is not understood, but has been used to effectively find some of the highest redshift radio galaxies.

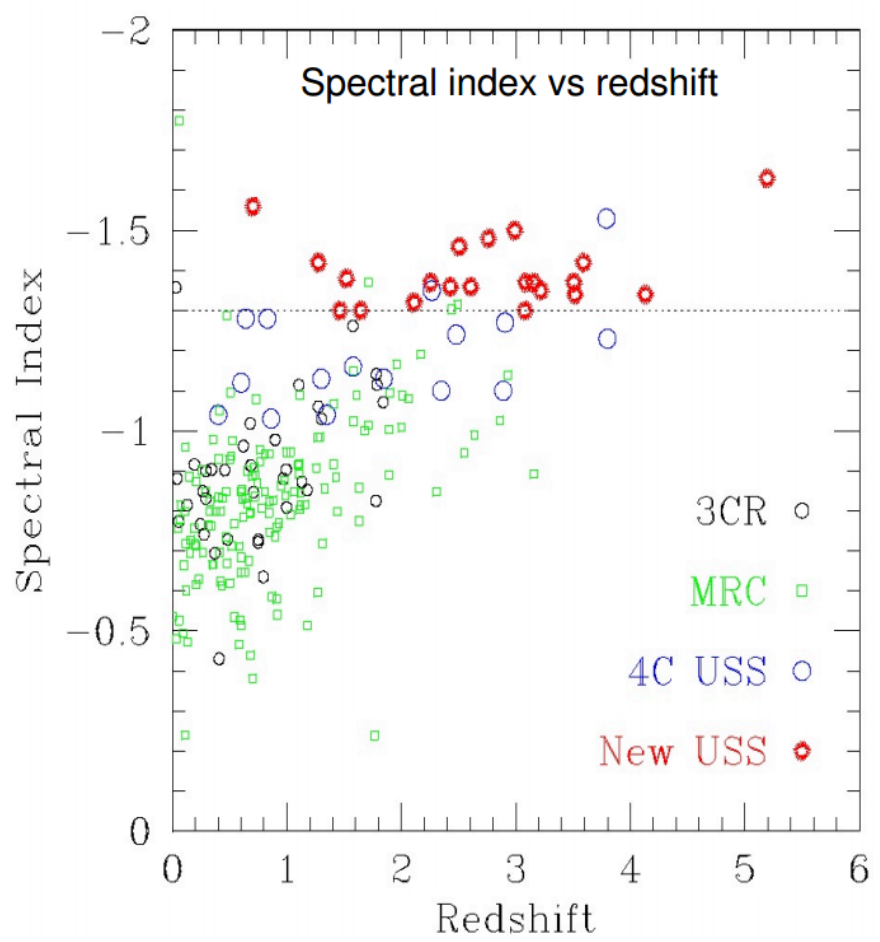

Figure 1: The empirical correlation between spectral index and redshift. Figure from De Breuck et al. (2000) 
Several explanations have been proposed for the $z-\alpha$ relation. One explanation is that the $z-\alpha$ relation is merely an observational consequence of limited observed frequency ranges. Low redshift radio galaxies exhibit curved spectra at the lowest frequencies. Any corresponding concavity in the rest-frame low-frequency spectra could be missing from observations because of the limited frequency range of radio instruments. Lower redshift sources also tend to steepen towards higher frequencies, and for fixed observing frequency ranges, higher redshift sources will be observed at higher rest-frame frequencies. This could also lead to an observed $z-\alpha$ relation, but when correcting the radio spectra to the rest-frame, the relation is still present. Another contribution to the steep spectra could come from inverse-Compton losses from the cosmic microwave background, which has an energy density dependent on $(1+z)^{4}$ (Krolik \& Chen, 1991). The observational effects and inverse-Compton losses are widely accepted in the literature as the main contributors to the $z-\alpha$ relation.

Another explanation is also an observational effect: Blundell \& Rawlings (1999) showed that the jet power is a link between luminosity and spectral index. The brighter a source is, the more powerful its jets are, and the stronger the magnetic fields are at the edges of the radio lobes. When observing objects at higher redshifts, only the brighter targets are observable, while fainter targets can be observed at low redshift. Thus a $z-\alpha$ relation could arise from a luminosity $-\alpha$ relation based on instrumental flux limits. However, the relation still persists even when taking flux-limited samples (Athreya \& Kapahi, 1998).

These explanations were investigated by Klamer et al. (2006). The authors collected the largest (at the time) sample of HzRGs with multi-frequency observations. They shifted all radio spectra to the rest-frame to show that the $k$-correction only partially explains the $z-\alpha$ relation. They also conclude that the luminosity $-\alpha$ relation is not the driving cause of the $z-\alpha$ relation. Instead, the authors suggest that the higher ambient density around HzRGs could cause steeper spectral indices as the jets plough through denser material than their low redshift counterparts.

Gathering information on HzRGs at low frequencies is crucial for resolving the cause of the $z-\alpha$ relation. Low frequency observations will reveal if HzRG ultra-steep spectra still remain ultrasteep at low frequencies, or if there is evidence for other physical mechanisms that would cause spectral turnovers. It is also important to determine what the spectral properties are on spatially resolved scales, as variation or similarity between the individual lobes of an object can provide environmental clues to help untangle the cause of the $z-\alpha$ relation. To this end, we have created a pilot survey of ultra-steep spectrum (USS) sources (HzRGs) with the Low Frequency Array (LOFAR), which will be described in the next section.

\section{The LOFAR Ultra Steep Spectrum Sources Survey}

We have chosen a sample of 10 USS sources and one low-redshift analogue (3C 368) that exhibits many of the same characteristics as its high redshift counterparts. The sources were initially chosen from all the known sources in the Northern hemisphere (see Figure 2) at $z>2$, with a spectral index steeper than $\alpha=1$. This sample was further refined by selecting objects that were expected to be brighter than $10 \mathrm{Jy}$ at $30 \mathrm{MHz}$ and have simple double-lobed morphology with angular sizes greater than $5^{\prime \prime}$. This ensures that there will be enough signal to noise for self-calibration, and 
ensures that we will be able to resolve the individual radio lobes. All targets have been observed with the LBA, and five of the targets have been observed with the HBA.

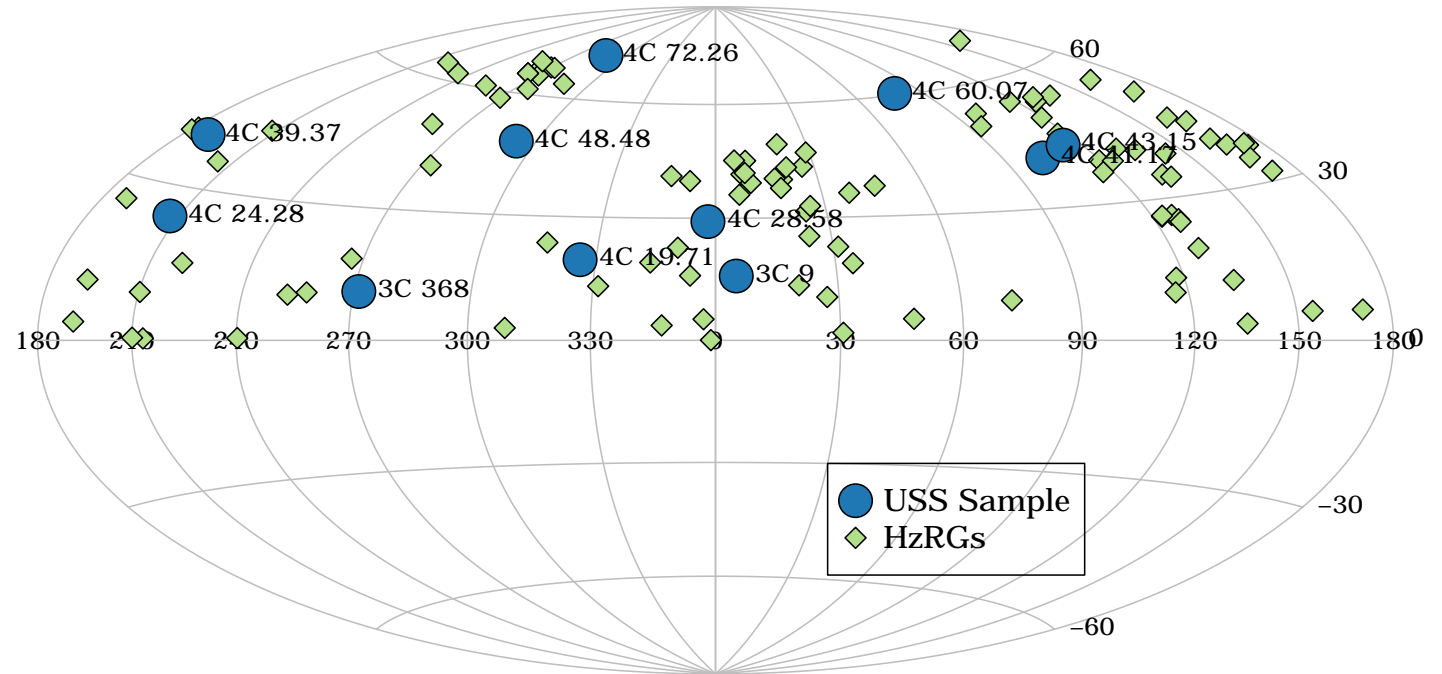

Figure 2: The USS sample sky distribution. Green diamonds are the location of all HzRGs in the Northern hemisphere listed in Miley \& De Breuck (2008). Blue circles show the sample.

\subsection{Goals}

This survey has three distinct goals:

1. Use only the Dutch stations of LOFAR for low resolution imaging to characterize the integrated low frequency spectra of USS sources.

2. By using the international baselines of LOFAR, study the spatially resolved spectra of USS sources to constrain the physical mechanisms responsible for their ultra-steep spectra. With resolutions of about $1^{\prime \prime}$ at $60 \mathrm{MHz}$, we will be able to:

- Determine the spatial location of the low- $v$ electrons

- Compare the radio lobes with each other as a probe of environmental conditions

3. Search USS sources for low-frequency Carbon radio recombination lines, which are a probe of the cold neutral medium. The first extragalactic detection of Carbon radio recombination lines was recently made using LOFAR observations of M82(Morabito et al., 2014). These types of sources are known to have large reservoirs of cold gas, making them ideal candidates for Carbon radio recombination line searches.

\subsection{Instruments}

This survey will make use of both the low band antenna (LBA) and high band antenna (HBA) of LOFAR. Table 1 lists pertinent information about both instruments. The LBA frequency range is critical to probe down to the lowest possible rest frequencies. The HBA provides: 


\begin{tabular}{lcc} 
& LBA & HBA \\
\hline Frequency Range & $10-90 \mathrm{MHz}$ & $120-240 \mathrm{MHz}$ \\
Resolution Dutch Array & $11^{\prime \prime}$ & $5^{\prime \prime}$ \\
Sensitivity Dutch Array & $866 \mu \mathrm{Jy} \mathrm{bm}^{-1}$ & $107 \mu \mathrm{Jy} \mathrm{bm}^{-1}$ \\
Resolution International Array & $1^{\prime \prime}$ & $0.5^{\prime \prime}$ \\
Sensitivity International Array & $608 \mu \mathrm{Jy} \mathrm{bm}^{-1}$ & $66 \mu \mathrm{Jy} \mathrm{bm}^{-1}$
\end{tabular}

Table 1: LOFAR instrument capabilities. Resolutions are listed for $60 \mathrm{MHz}$ and $150 \mathrm{MHz}$ for the LBA and HBA, respectively. Sensitivities were calculated for $15 \mathrm{MHz}$ of bandwidth, for an $8 \mathrm{hr}$ observation, at $60 \mathrm{MHz}$ and $150 \mathrm{MHz}$.

- Morphological information closer in frequency and resolution to the LBA; this can provide excellent models to use for self-calibration.

- Extended frequency coverage for Carbon radio recombination line searches.

For the LBA portion of the survey the available bandwidth was split evenly into two simultaneous beams, one on the primary calibrator and one on the target. This provided $48 \mathrm{MHz}$ of bandwidth from $30-78 \mathrm{MHz}$ on each object. The HBA is limited to a separation of about 15 degrees for multiple beams, and thus we observe primary calibrators at the beginning and end of each observation.

\section{Data Reduction}

\subsection{Low Resolution Imaging: LBA}

The low resolution imaging is performed by using only the Dutch stations of LOFAR. All USS sources will be unresolved by the Dutch array. We transfer calibration solutions from the calibrator to the target. We perform a phase-only calibration on the target field to solve for differential ionospheric and beam effects. An example resulting image is shown in Figure 3 for the target $4 \mathrm{C}$ +39.37 .

\subsection{High Resolution Imaging}

Making high resolution imaging at low frequencies is challenging for a number of reasons. Correlating data for the long baselines of LOFAR (up to $1300 \mathrm{~km}$ ) requires accurate geometric models, which will have residual errors. This is complicated by the fact that while the inner core LOFAR stations are all on the same clock, the remote and international stations have individual clocks. This leads to clock offsets, which can cause delay errors as well. Other issues are particular to low frequencies. The total electron content in the ionosphere depends on has a $v^{-2}$, and hence becomes much stronger at lower frequencies. Ionospheric conditions can be completely different for the international LOFAR stations, making it difficult to reconstruct the signal, especially for the LBA. Techniques that optimize signal to noise to solve for residual delays, rates, and phases are necessary. One final issue is the lack of known calibrator sources at low frequencies, which we have attempted to overcome by choosing bright targets that are suitable for self-calibration. 


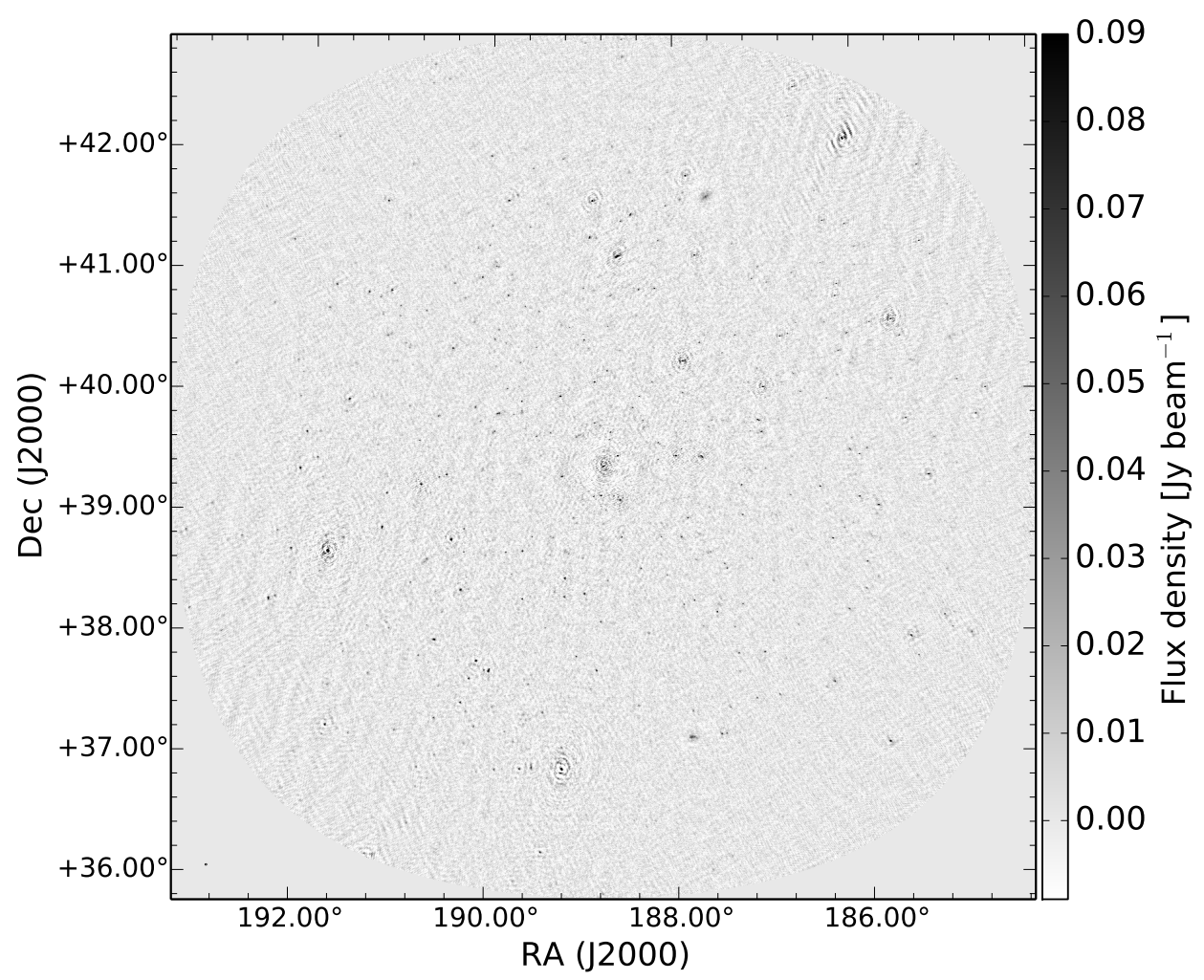

Figure 3: An example wide-field image for target $4 \mathrm{C}+39.37$. This image was made with $1.95 \mathrm{MHz}$ of bandwidth, a beam size of approximately $15^{\prime \prime}$, rms noise of $7.5 \mathrm{mJy} \mathrm{bm}^{-1}$, and a 7 degree field of view.

All of these issues lead us to use traditional very long baseline interferometry (VLBI) techniques. Specifically, we use the global fringe-fitting algorithm in the Astronomical Image Processing System (AIPS). There is now a standard observatory pipeline that prepares LOFAR HBA long baseline data for AIPS by correcting the data with beam models, phasing up the core stations and combining them into a single 'super' station, and converting the measurement sets to AIPS uvfits format. The data reduction for LBA long baseline data is still being finalized via this USS survey, and requires additional amplitude corrections from the primary calibrator. This will be the topic of a future paper (Morabito et al., in preparation).

\section{Preliminary Results}

\subsection{Low Resolution Imaging}

Figure 4 shows the integrated spectra for two of the targets. One of these targets shows a straight, steep spectrum all the way down to $100 \mathrm{MHz}$ (rest-frame), while the other target shows a turnover in the spectrum by $100 \mathrm{MHz}$ (rest-frame). The variation in targets is perhaps indicative of an environmental effect, but the complete USS survey sample is necessary to begin identifying trends. 

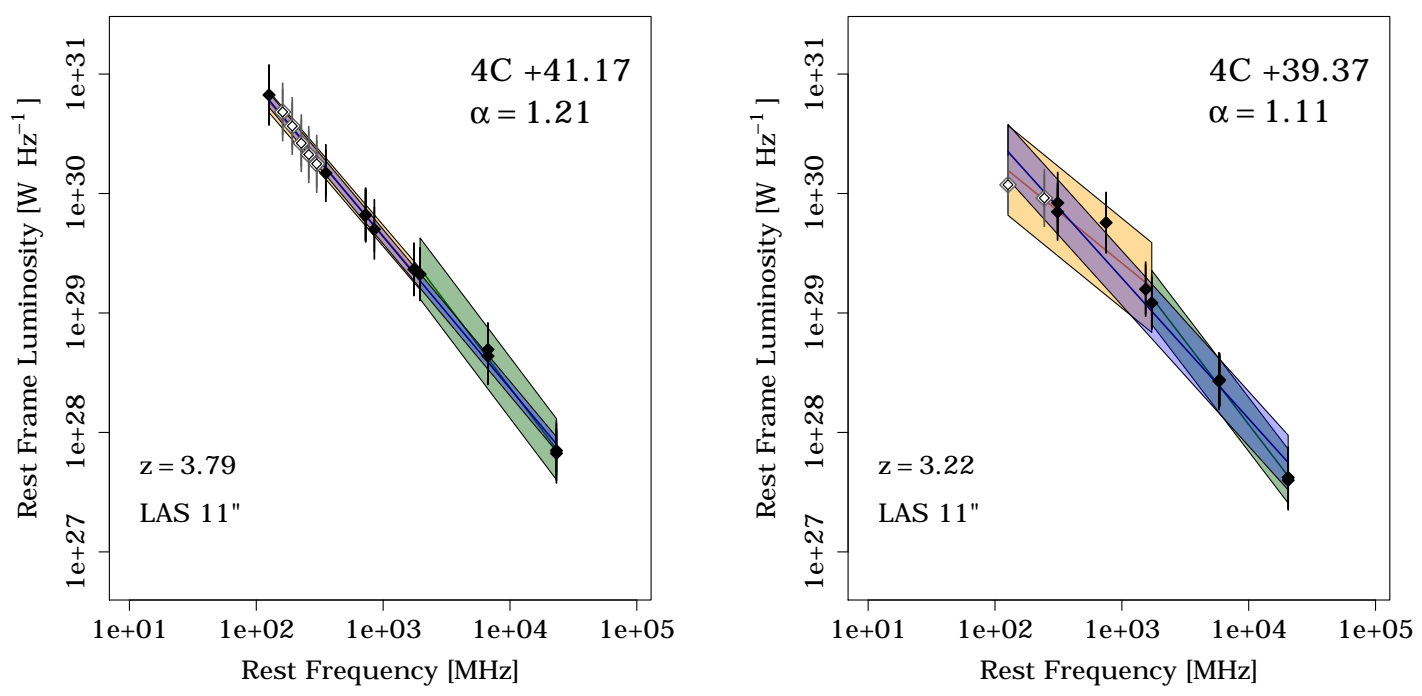

Figure 4: Preliminary results from low resolution imaging. The rest-frame integrated spectra are shown for two targets. Black diamonds are from archival observations, and white diamonds are the new LOFAR measurements. The shaded areas represent the errors on power-law fits to the spectra: blue is for a single power-law fit to all data; orange is a single power-law fit to the low frequency portion of the spectra; and green is a single power-law fit to the high frequency portion of the spectra. Deviations between the blue fit and the green and orange fits indicate spectral curvature.

\subsection{High Resolution Imaging}

Here we provide preliminary results for the HBA high resolution imaging. The LBA high resolution imaging will be discussed in a forthcoming paper (Morabito et al., in preparation). Figure 5 shows an initial, un-self-calibrated image of $4 \mathrm{C}+39.37$ as seen by the HBA. This image shows the same lobe-like morphology as seen at higher frequencies. This indicates that the low and high frequency electron populations are spatially linked. Extracting qualitative spectral index information from these images requires further self-calibration.

\section{Summary}

The preliminary results from this LOFAR survey of USS sources are:

- The integrated spectra of two of the targets show variety.

- The morphology of USS sources at low frequencies are similar to the morphology at high frequencies.

These preliminary results are based on only two objects, and complete processing of the USS Survey is necessary to start identifying general trends. Although the survey only has 11 targets, this will be enough to (a) sort out calibration issues and streamline the data reduction and (b) show any general trends, or lack thereof, to guide future, larger surveys.

The authors are working on finalizing the LBA high resolution data reduction, which is the last necessary step before completing the data reduction of all observed targets. 

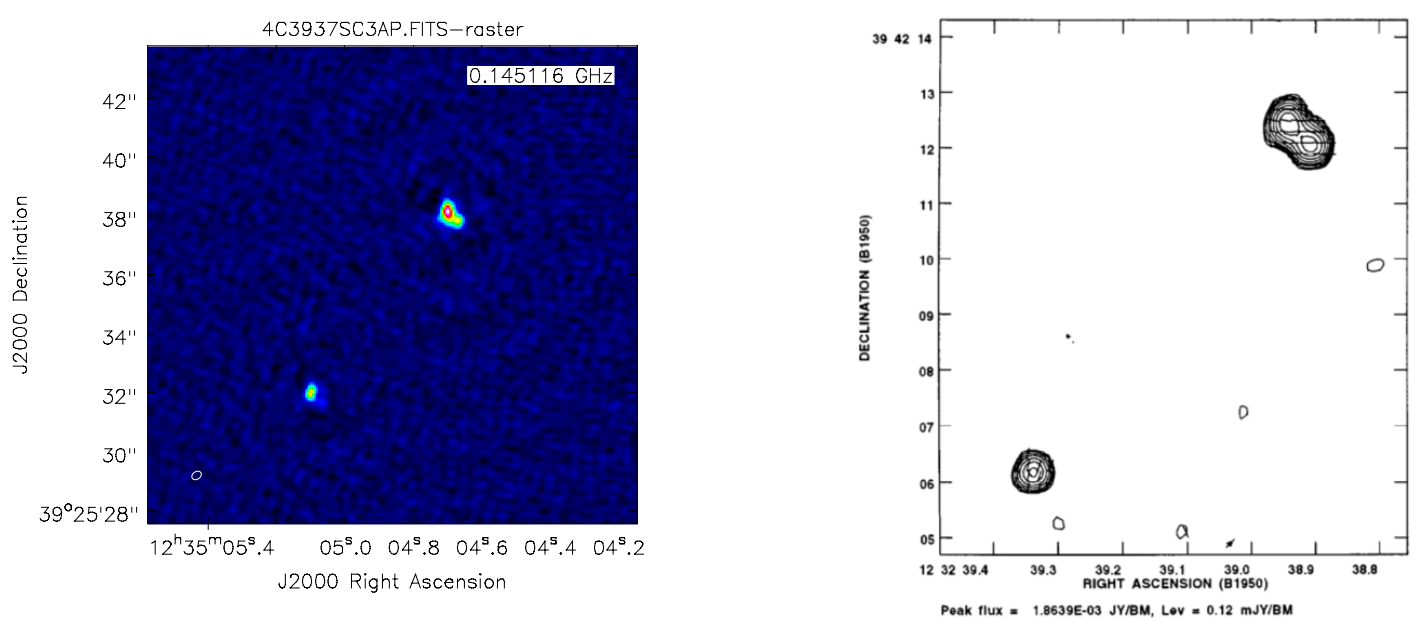

Figure 5: Left: The target $4 \mathrm{C}+39.37$ as seen by LOFAR at $145 \mathrm{MHz}$. Right: $4 \mathrm{C}+39.37$ at $4 \mathrm{GHz}$ with the Very Large Array (Carilli et al., 1997).

\section{References}

Athreya, R. M., \& Kapahi, V. K. 1998, Journal of Astrophysics and Astronomy, 19, 63

Bicknell, G. V., Sutherland, R. S., van Breugel, W. J. M., et al. 2000, ApJ, 540, 678

Blumenthal, G., \& Miley, G. 1979, A\&A, 80, 13

Blundell, K. M., \& Rawlings, S. 1999, Nature, 399, 330

Carilli, C. L., Röttgering, H. J. A., van Ojik, R., et al. 1997, ApJS, 109, 1

Chambers, K. C., Miley, G. K., \& van Breugel, W. 1987, Nature, 329, 604

Cooke, E. A., Hatch, N. A., Muldrew, S. I., Rigby, E. E., \& Kurk, J. D. 2014, MNRAS, 440, 3262

De Breuck, C., van Breugel, W., Röttgering, H. J. A., \& Miley, G. 2000, A\&A Supp., 143, 303

Klamer, I. J., Ekers, R. D., Bryant, J. J., et al. 2006, MNRAS, 371, 852

Krolik, J. H., \& Chen, W. 1991, AJ, 102, 1659

Kurk, J. D., Röttgering, H. J. A., Pentericci, L., et al. 2000, A\&A, 358, L1

Miley, G., \& De Breuck, C. 2008, A\&A Rev., 15, 67

Morabito, L. K., Oonk, J. B. R., Salgado, F., et al. 2014, ApJ, 795, L33

Pentericci, L., Röttgering, H. J. A., Miley, G. K., et al. 1999, A\&A, 341, 329

Tielens, A. G. G. M., Miley, G. K., \& Willis, A. G. 1979, A\&A Supp., 35, 153 